\title{
Correction: TERT promoter mutation in adult granulosa cell tumor of the ovary
}

\author{
Jessica A. Pilsworth ${ }^{1,2}$ - Dawn R. Cochrane ${ }^{2}$ Zhouchunyang Xia ${ }^{2,3}$ - Geraldine Aubert ${ }^{4}$ Anniina E. M. Färkkilä5,6 • \\ Hugo M. Horlings ${ }^{2,3}$. Satoshi Yanagida ${ }^{7}$ Winnie Yang ${ }^{2}$ - Jamie L. P. Lim $^{2} \cdot$ Yi Kan Wang ${ }^{2}$. Ali Bashashati ${ }^{2}$. \\ Jacqueline Keul $^{8} \cdot$ Adele Wong $^{10} \cdot$ Kevin Norris $^{11} \cdot$ Sara Y. Brucker $^{8} \cdot$ Florin-Andrei Taran $^{8} \cdot$ Bernhard Krämer $^{8}$. \\ Annette Staebler ${ }^{12} \cdot$ Hannah van Meurs ${ }^{9}$ - Esther Oliva ${ }^{10}$ - Sohrab P. Shah $\mathbb{D}^{2,13} \cdot$ Stefan Kommoss ${ }^{8}$. \\ Friedrich Kommoss $^{14}$ - C. Blake Gilks ${ }^{3}$ Duncan M. Baird ${ }^{11}$ - David G. Huntsman ${ }^{2,3}$
}

Published online: 3 July 2018

(c) United States \& Canadian Academy of Pathology 2018

Correction to: Modern Pathology https://doi.org/10.1038/ s41379-018-0007-9; published online 15 February 2018

The original version of this article omitted the author Hannah van Meurs from the Department of Gynecology,
Center for Gynecologic Oncology Amsterdam, Academic Medical Center, 1100 DD Amsterdam, The Netherlands.

This has been corrected in both the PDF and HTML versions of the article.
David G. Huntsman

dhuntsma@bccancer.bc.ca

1 Department of Medical Genetics, University of British Columbia, Vancouver, BC, Canada

2 Department of Molecular Oncology, British Columbia Cancer Agency, Vancouver, BC, Canada

3 Department of Pathology and Laboratory Medicine, University of British Columbia, Vancouver, BC, Canada

4 Terry Fox Laboratory, British Columbia Cancer Agency, Vancouver, BC, Canada

5 Children's Hospital and Department of Obstetrics and Gynecology, University of Helsinki and Helsinki University Hospital, Helsinki, Finland

6 Department of Radiation Oncology, Harvard Medical School, Dana-Farber Cancer Institute, Boston, MA, USA

7 Department of Obstetrics and Gynecology, The Jikei University
School of Medicine, Tokyo, Japan

8 Department of Women's Health, Tübingen University Hospital, Tübingen, Germany

9 Department of Gynecology, Center for Gynecologic Oncology Amsterdam, Academic Medical Center, 1100 DD, Amsterdam, The Netherlands

10 Department of Pathology, Massachusetts General Hospital, Boston, MA, USA

11 Division of Cancer and Genetics, School of Medicine, Cardiff University, Cardiff, UK

12 Tübingen University Hospital, Institute of Pathology, Tübingen, Germany

13 Department of Computer Science, University of British Columbia, Vancouver, BC, Canada

14 Institute of Pathology, Campus Bodensee, Friedrichshafen, Germany 